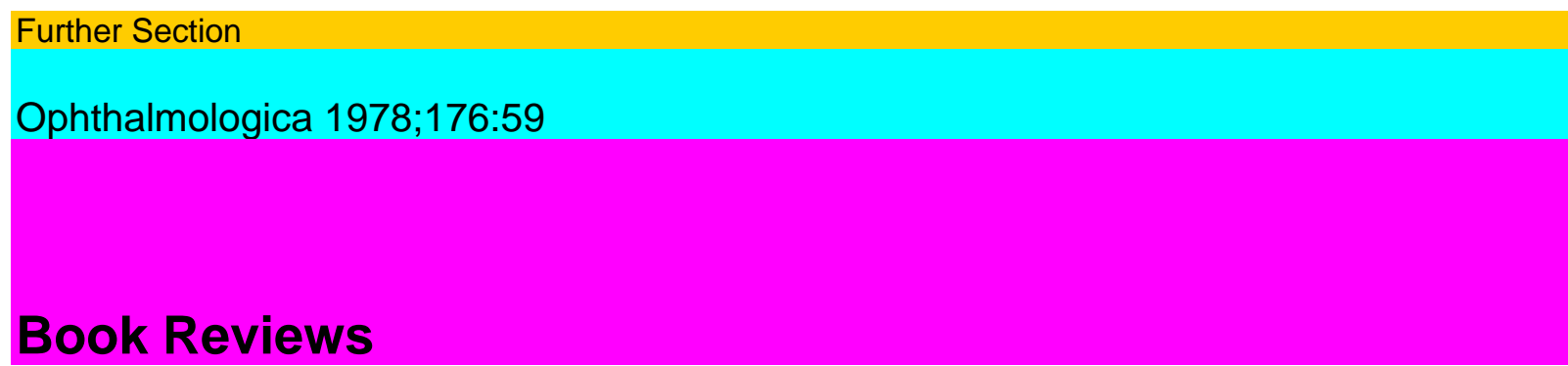

· Buchbesprechungen · Livres nouveaux

A. R. Irvine and C. O'Malley feds.): Advances in vitreous Surgery

Thomas, Springfield 1976720 pp.; \$51.-1SBN 0-398-03391-9

De nouvelles techniques et une instrumentation appropriée ont permis ces dernières années le développement d'une chirurgie touchant le vitré con-sidéré longtemps comme inaccessible.

Cet ouvrage est une mise en commun des con-naissances et des experiences accumulees par les maîtres de cette chirurgie aux Etats-Unis et en Europe.

«La structure du vitré, Гexamen morphologique et fonctionnel du patient souffrant d'une affection du vitré, les techniques de vitrectomie < à ciel ou-vert $>$ ou par la pars plana, Гusage de la diathermie et des ultrasons dans la loge vitréenne, sont exposes au fil des chapitres traitant de problèmes spécifiques tels que décollement de rétine, rétinopathie diabé-tique ou traumatisme.

On y trouve à la fois des experiences cliniques pratiques et toutes sortes de suggestions

intéressantes.»

La somme de ces travaux reflète bien la situation actuelle de la chirurgie vitréenne et nous pa-raît indispensable à ceux qui s'intéressent à ce type de chirurgie.

Ce livre permettra aussi au praticien de sélec-tionner les patients qui pourront bénéficier des progrès de cette chirurgie vitréenne en plein essor.

W. D. Schäfer

Strabismus in der Praxis. Untersuchungstechnik

und Behandlungsablauf

Springer, Berlin 1976

XI+ 137 pp., 37 fig.; DM 18.80

ISBN 3-540-07782-0

Eine kurze Einführung und Übersicht auf die Behandlungsmethodik einer Sehschule wird in diesem Bändchen präsentiert. Bewusst wird auf dieBe-schreibung spezieller Schielformen und auf diagno-stische Einzelheiten verzichtet, aber auf einschlä-gige Literatur hingewiesen.

Dieses Taschenbuch darf alien orthoptisch ln-teressierten zur Einführung, Schnellorientierung oder als Auffrischkurs empfohlen werden.

\title{
S. Stress
}

Lausanne

A. Balmer

Lausanne 